\title{
Clinical Profile and Treatment Outcome of Culture-negative Infective Endocarditis in Children at UP-PGH: A Ten-year Retrospective Study
}

\author{
Mylah P. Alfeche ${ }^{1}$ and Ma. Concepcion S. Sison ${ }^{1}$ \\ ${ }^{1}$ Section of Cardiology, Department of Pediatrics, College of Medicine and Philippine General Hospital, University of the Philippines Manila
}

\begin{abstract}
Objective. This study determined the demographic data, clinical profile, treatment and outcome of BCN-IE in children at UP-PGH.

Methods. This is a retrospective study of children with BCN-IE admitted at UP-PGH from 2004-2013. Demographic data, clinical presentation, previous antibiotic use, echocardiographic findings, an antibiotic used and outcome of patients were recorded and analyzed. Results in the demographic and clinical profile were expressed as frequencies, percentages and means. To compare the clinical features, echocardiographic findings and antibiotic regimen used as to the outcome, Fischer's exact test was used.
\end{abstract}

Results. Among 91 patients diagnosed with infective endocarditis, 61.54\% had BCN-IE. The age, gender and clinical presentation were similar to other studies. Clinical presentation and echocardiographic findings did not have influence on outcome of children with BCN-IE. The use of penicillin $\mathrm{G}$ and amikacin is associated with unfavorable outcome after 4 weeks or less of administration.

Conclusion. The incidence of BCN-IE is high in UP-PGH. The demographic distribution, clinical and echocardiographic features of patients do not have an influence on the outcome. The trend to the unfavorable outcome with the use of penicillin suggests the need to target fastidious organisms in BCN-IE. Further investigation is warranted to establish the etiologic agents of BCN-IE.

Key Words: culture-negative endocarditis, children, outcome

Presented at the 7th World Congress of Pediatric Cardiology and Cardiac Surgery on July 16-21, 2017, Barcelona, Spain.

Awarded 2nd place in Oral presentation at the Department of Pediatrics Fellows' Oral Presentation Forum on November 4, 2015 at the University of the Philippines Manila - Philippine General Hospital, Philippines.

Corresponding author: Mylah P. Alfeche, MD

Section of Cardiology

Department of Pediatrics

College of Medicine

Philippine General Hospital

University of the Philippines Manila

Taft Avenue., Manila 1000, Philippines

Telephone: +6325548400 local 2125

Email:mylahrmt@yahoo.com

\section{BACKGROUND}

Infective endocarditis (IE) is an infrequent but serious disease in infants and children. It is associated with considerable morbidity and mortality and remains a dreaded complication of structural heart disease. It is a microbial infection of the endocardial (endothelial) surface of the heart, which may include one or more heart valves, the mural endocardium, septal defects, or intravascular foreign devices. ${ }^{1}$ It remains a diagnostic and therapeutic challenge. It is usually suspected in a patient with fever and a new or changing cardiac murmur. Currently, IE is diagnosed based on Duke's criteria which are a combination of clinical, echocardiographic and laboratory parameters, most often by presence of vegetations on echocardiogram and positive blood culture. ${ }^{2}$ Nevertheless, a negative blood culture does not rule out the possibility of IE.

Targeted antibiotic treatment is the ideal approach to the pharmacological management of IE, so the identity 
of the pathogen causing the disease must be determined whenever possible. A diagnosis of culture-negative endocarditis is made when a patient has clinical and/or echocardiographic evidence of IE but persistently negative blood cultures. Blood cultures can be negative in $\leq 20 \%$ of cases of endocarditis. ${ }^{13-5}$ Factors that influence the rate of culture negativity in infective endocarditis include failure to use appropriate culture technology, the use of antibiotics before the collection of blood culture samples, and infection caused by fastidious or non-culturable pathogens. ${ }^{6-10}$ Based on the American Heart Association and European Society of Cardiology guidelines, blood culture-negative infective endocarditis (BCN-IE) must be treated with antibiotics targeting fastidious organisms. ${ }^{3,11}$

Infective endocarditis is a complex disease to treat, made even more challenging in patients who have negative blood cultures. The importance lies in the significant morbidity and mortality associated with the disease, the need for selecting appropriate parenteral antibiotic treatment and identifying potential complications from the embolic and immunologic phenomenon. At present, there is no substantial description of demographic and clinical profile of BCN-IE in children in the country. Patients diagnosed with IE upon admission are given empiric antibiotics based on the American Heart Association guidelines. The antibiotic use and outcome in this population needs to be reviewed. Whether BCN-IE responds to a routine treatment regimen for IE remains to be seen. This study determined the demographic data, clinical profile, treatment and outcome of $\mathrm{BCN}-\mathrm{IE}$ in children admitted at UP-PGH.

\section{METHODS}

This is a retrospective study of children aged 0-18 years old admitted at the University of the Philippines Philippine General Hospital (UP-PGH) from 2004 to 2013 who were diagnosed with BCN-IE. A clinical diagnosis of definite IE was based on modified Duke's criteria (Table 1). Patients diagnosed with definite IE with persistent negative blood cultures were included in the study. Those with incomplete medical records were excluded. Patients' age, weight, length/height, body mass index (BMI) and gender were recorded in the demographic data. Their clinical presentation, namely, symptom(s) and its onset at the time of admission, NYHA functional classification (FC), the presence of predisposing heart disease and history of previous antibiotic use for the current symptom(s), were also noted. The echocardiographic findings specifically the presence of oscillating mass, location and size of vegetation(s), the presence of cardiac abscess and valvular dysfunction were documented. Most importantly, the antibiotics used and outcome of the patients were recorded. Patients who were discharged after completion of treatment were classified to have favorable outcome. On the other hand, those who went home against medical advice (HAMA), died during admission and was readmitted one month after discharge, are classified to have an unfavorable outcome. Records for the study were anonymized and kept confidential. There was no conflict of interest in any form - financial, proprietary and professional.

Results in the demographic and clinical profile of the patients were expressed as frequencies, percentages and

Table 1. Modified Duke Criteria for the Diagnosis of Infective Endocarditis (IE) ${ }^{2}$

Major Criteria

1. Positive blood culture for IE

a. Typical microorganism consistent with IE from 2 separate blood cultures as noted below:

i. viridians streptococci, Streptococcus bovis, or HACEK group, or

ii. community-acquired Staphylococcus aureus or enterococci, in the absence of a primary focus, or

b. Microorganisms consistent with IE from persistently positive blood cultures defined as

i. $\quad \geq 2$ positive blood cultures drawn $>12$ hours apart or

ii. all of 3 or a majority of $\geq 4$ separate blood cultures (with first and last sample drawn $\geq 1$ hour apart)

2. Evidence of endocardial involvement

a. Positive echocardiogram for IE defined as

i. Oscillating intracardiac mass on the valve or supporting structures, in the path of regurgitant jets, or on implanted material in the absence of an alternative anatomic explanation, or

ii. abscess, or

iii. new partial dehiscence of prosthetic valve, or

b. New valvular regurgitation (worsening or changing of preexisting murmur not sufficient)

Minor Criteria

1. Predisposition: predisposing heart condition or intravenous drug use

2. Fever: temperature $\geq 38.0 \mathrm{C}$

3. Vascular phenomena: major arterial emboli, septic pulmonary infarcts, mycotic aneurysm, intracranial hemorrhage, conjunctival hemorrhages, and Janeway lesions

4. Immunologic phenomena: glomerulonephritis, Osler's nodes, Roth spots and rheumatoid factor

5. Microbiological evidence: positive blood culture but does not meet a major criterion as noted above or serological evidence of active infection with an organism consistent with IE

6. Echocardiographic findings: consistent with IE but do not meet a major criterion as noted above

Clinical Criteria of Definite IE:

1. 2 major criteria; or

2. 1 major criterion and 3 minor criteria;

3. or 5 minor criteria 
means. In comparing the clinical features, echocardiographic findings and antibiotic regimen used to the outcome of patients, Fischer's exact test was used with alpha $=0.05$ as the level of significant statistical difference.

\section{RESULTS}

A total of 91 pediatric patients diagnosed with infective endocarditis were admitted at UP-PGH from 2004-2013. Among these patients, 56 (61.54\%) were included in the study because they showed negative results in their blood culture studies upon admission. The youngest was 2 weeks old and the oldest was 18 years old (mean $10.15 \pm 4.83$ years). Majority (44.64\%) of these patients were in their early teenage years. The mean weight and length/height were $25.65 \pm 11.34 \mathrm{~kg}(1.3-46.3 \mathrm{~kg})$ and $127.66 \pm 26.07$ $\mathrm{cm}(45-169 \mathrm{~cm})$, respectively. Body mass index ranged from $6.4 \mathrm{~kg} / \mathrm{m}^{2}$ to $22.9 \mathrm{~kg} / \mathrm{m}^{2}$ (mean $\left.=14.76 \pm 2.82 \mathrm{~kg} / \mathrm{m}^{2}\right)$. Twenty nine (51\%) patients were female and 27 (48\%) were male. (Table 2)

The most frequent clinical presentation upon admission was fever (89.29\%). The second most common presentation was dyspnea/orthopnea (46.43\%) followed by easy fatigability (32.15\%), cough (30.36\%) and edema (23.21\%). Nine (16.07\%) patients required oxygen supplementation and $9(16.07 \%)$ presented with anorexia. Pallor and headache were seen in $7(12.5 \%)$ patients. Six (10.71\%) patients had hemiparesis, and $5(8.93 \%)$ had hepatomegaly. Other clinical presentations include increased sleeping time, seizure, joint pain, jaundice, abdominal pain, body malaise, hematuria, new-onset/changing murmur, vomiting, hemoptysis/hematemesis, chest pain and diarrhea. (Table 3)

\section{Onset of Symptoms and Functional Status}

Majority (76.79\%) of the patients had symptoms for more than 2 weeks prior to admission, while $23.21 \%$ had symptoms for less than or equal to 2 weeks. Fifty percent were in either NYHA functional class III or IV, while 30\% were in functional class II. The remaining $20 \%$ of patients were in functional class I. (Table 3)

\section{Predisposing Heart Disease}

Most of the patients had predisposing congenital or acquired heart disease. Only 3 out of 56 had no predisposing heart disease. These three were aged 2 weeks, 9 and 13 years old. Twenty five (42.86\%) patients had acyanotic heart disease in which VSD (25\%) was the most common, followed by PDA (14.28\%), ASD (3.57\%) then congenital MR (1.79\%). Nine (10.86\%) patients had cyanotic heart disease in which TOF (8.93\%) was the most common followed by DORV (3.57\%), DORV-PVA (1.79\%) and PVA-VSD (1.79\%). Among the subjects, 19 (33.93\%) had rheumatic fever/rheumatic heart disease. (Table 4)

\section{Previous Antibiotic Use}

Thirteen (23.21\%) patients had been given antibiotics prior to admission. Antibiotics taken were amoxicillin, ampicillin, amikacin, cotrimoxazole, cephalexin, cefuroxime, cefaclor, cefazolin, ceftazidime, and penicillin G. On the other hand, more than three fourths $(76.79 \%)$ had no previous antibiotic intake prior to the admission. (Table 3)

\section{Echocardiographic Findings}

All subjects had oscillating mass/vegetation echocardiographically. Six (10.71\%) patients had vegetations on multiple locations, while the rest of the patients had solitary cardiac structural involvement in which mitral valve (37.5\%) was most commonly involved followed by the tricuspid valve (8.93\%). The RVOT, pulmonary valve and aortic valve (native) were equally affected in frequency (7.14\%). The rest of the patients had vegetations located solely at the pulmonary artery, right atrium, left atrium, interatrial septum or interventricular septum. One patient had vegetation at the prosthetic aortic valve. Most (37.5\%) of the patients had vegetation sizes of less than $5 \mathrm{~mm}$. About a third of the subjects had vegetation sizes of more than 10 $\mathrm{mm}$. More than half of the subjects (58.93\%) had multiple valvular dysfunctions. Mitral regurgitation was the most common and can be seen in $90 \%$ of the subjects. Both mitral regurgitation and aortic regurgitation were seen in 6 (10.71\%) patients. (Table 5)

Table 2. Demographic data of patients with $B C N$-IE

\begin{tabular}{lccc} 
Characteristics & Range $($ Mean \pm SD) & No. of patients & Percentage $(\mathbf{n}=56)$ \\
Age (in years) & $0.04-18(10.15 \pm 4.83)$ & & - \\
$<2$ & & 2 & 3.57 \\
$2-<3$ & & 2 & 3,57 \\
$3-<6$ & 5 & 8.93 \\
$6-<12$ & & 22 & 39.29 \\
$12-18$ & & & 44.64 \\
Weight $(\mathrm{kg})$ & $1.3-46.3(25.65 \pm 11.34)$ & & - \\
Length/Height $(\mathrm{cm})$ & $45-169(127.66 \pm 26.07)$ & & - \\
BMI $\left(\mathrm{kg} / \mathrm{m}^{2}\right)$ & $6.4-22.9(14.76 \pm 2.82)$ & & - \\
Sex & & 27 & 48.21 \\
$\quad$ Male & & 29 & 51.79 \\
Female & & & \\
\hline
\end{tabular}


A Ten-year Review of Profile and Outcome of BCN-IE in UP-PGH

Table 3. Clinical presentation of patients with BCN-IE

\begin{tabular}{|c|c|c|c|}
\hline Characteristics & & No. of patients & Percentage $(n=56)$ \\
\hline Presentation & Fever & 50 & 89.29 \\
\hline & Dyspnea/Orthopnea & 26 & 46.43 \\
\hline & Easy fatigability & 18 & 32.14 \\
\hline & Cough & 17 & 30.36 \\
\hline & Edema & 13 & 23.21 \\
\hline & Anorexia & 9 & 16.07 \\
\hline & $\mathrm{O}_{2}$ support & 9 & 16.07 \\
\hline & Pallor & 7 & 12.5 \\
\hline & Headache & 7 & 12.5 \\
\hline & Hemiparesis & 6 & 10.71 \\
\hline & Hepatomegaly & 5 & 8.93 \\
\hline & Increased sleeping time & 4 & 7.14 \\
\hline & Seizure & 4 & 7.14 \\
\hline & Joint pains & 3 & 5.36 \\
\hline & Jaundice & 3 & 5.36 \\
\hline & Abdominal pain & 2 & 3.57 \\
\hline & Body malaise & 2 & 3.57 \\
\hline & Hematuria & 2 & 3.57 \\
\hline & Murmur & 2 & 3.57 \\
\hline & Vomiting & 1 & 1.79 \\
\hline & Hemoptysis/Hematemesis & 1 & 1.79 \\
\hline & Chest pain & 1 & 1.79 \\
\hline & Diarrhea & 1 & 1.79 \\
\hline Onset of symptoms & $\leq 2$ weeks & 13 & 23.21 \\
\hline & $>2$ weeks & 43 & 76.79 \\
\hline Functional Class on admission & Class I & 11 & 19.64 \\
\hline & Class II & 17 & 30.36 \\
\hline & Class III & 14 & 25 \\
\hline & Class IV & 14 & 25 \\
\hline Previous antibiotic use for current symptoms & Yes & 13 & 23.21 \\
\hline & No & 43 & 76.79 \\
\hline
\end{tabular}

Table 4. Predisposing heart disease of patients with BCN-IE

\begin{tabular}{llcc} 
& Predisposing Heart Disease & No. of patients & Percentage $(\mathbf{n = 5 6})$ \\
Acyanotic & VSD & 14 & 25 \\
& PDA & 8 & 14.28 \\
& ASD & 2 & 3.57 \\
\hline Cyanotic & Congenital MR & 1 & 1.79 \\
& TOF & 5 & 8.93 \\
& DORV & 2 & 3.57 \\
& DORV-PVA & 1 & 1.79 \\
\hline Acquired heart disease & PVA-VSD & 1 & 1.79 \\
\hline None & RF/RHD & 19 & 33.93 \\
\hline
\end{tabular}

\section{Outcome}

Most (73.21\%) of the patients were discharged improved after treatment. (Table 6) Among 38 patients given Penicillin $\mathrm{G}$ and Amikacin, 11 had an unfavorable outcome. Five died on or before 4 weeks of antibiotic. The causes of death include myocardial failure, low cardiac output syndrome and cardioembolic stroke. Of the three patients who went home against medical advice, one had a cardioembolic stroke, one had congestive heart failure (FC IV), and the other one was brought home with unknown reason. A patient without known heart disease, who had vegetation at the mitral valve with severe mitral regurgitation, was readmitted within a month after 4 weeks of treatment. Two patients who had rheumatic heart disease with cardioemblic stroke went home against medical advice after receiving 6 weeks of penicillin $\mathrm{G}$ and amikacin. Among 3 patients who received penicillin $G$, oxacillin and amikacin, 2 died on the fourth week of treatment. The causes of death were a myocardial failure and septic shock. A patient who had a cardioembolic stroke, S/P PDA transection, went home against medical advice. Among 4 patients given ceftazidime and amikacin, only 1 died due to cardioembolic stroke after 6 weeks of 
Table 5. Echocardiographic findings in patients with BCN-IE

\begin{tabular}{|c|c|c|c|}
\hline \multicolumn{2}{|c|}{ Characteristics } & No. of patients & Percentage $(n=56)$ \\
\hline \multicolumn{2}{|c|}{ Presence of oscillating mass/vegetation } & 56 & 100 \\
\hline \multirow[t]{12}{*}{ Location of vegetation } & Mitral Valve & 21 & 37.5 \\
\hline & Tricuspid Valve & 5 & 8.93 \\
\hline & RVOT & 4 & 7.14 \\
\hline & Pulmonary Valve & 4 & 7.14 \\
\hline & Aortic Valve (native) & 4 & 7.14 \\
\hline & Pulmonary Artery & 3 & 5.36 \\
\hline & Right Atrium & 2 & 3.57 \\
\hline & Left Atrium & 2 & 3.57 \\
\hline & Interatrial Septum & 2 & 3.57 \\
\hline & Interventricular septum & 2 & 3.57 \\
\hline & Aortic Valve (Prosthetic) & 1 & 1.79 \\
\hline & Multiple Locations & 6 & 10.71 \\
\hline \multirow[t]{3}{*}{ Size of vegetation } & $<5 \mathrm{~mm}$ & 21 & 37.5 \\
\hline & $5-10 \mathrm{~mm}$ & 16 & 28.57 \\
\hline & $>10 \mathrm{~mm}$ & 19 & 33.93 \\
\hline Cardiac abscess & & 0 & - \\
\hline \multirow[t]{12}{*}{ Valvular dysfunction } & Solitary & & \\
\hline & $\mathrm{MR}$ & 3 & 5.36 \\
\hline & $A R$ & 3 & 5.36 \\
\hline & TR & 3 & 5.36 \\
\hline & $\mathrm{PR}$ & 1 & 1.79 \\
\hline & Multiple & 33 & 58.93 \\
\hline & $\mathrm{MR}, \mathrm{AR}, \mathrm{TR}$ & 10 & 17.86 \\
\hline & $\mathrm{MR}, \mathrm{TR}$ & 9 & 16.07 \\
\hline & $M R, A R$ & 6 & 10.71 \\
\hline & $\mathrm{MR}, \mathrm{AR}, \mathrm{TR}, \mathrm{PR}$ & 4 & 7.14 \\
\hline & $\mathrm{AR}, \mathrm{TR}$ & 3 & 5.36 \\
\hline & $\mathrm{MR}, \mathrm{TR}, \mathrm{PR}$ & 1 & 1.79 \\
\hline
\end{tabular}

Table 6. Outcome of BCN-IE

\begin{tabular}{lccc} 
& Outcome & No. of patients & Percentage $(\mathbf{n}=\mathbf{5 6})$ \\
\hline Favorable & Discharged & 41 & 73.21 \\
\hline Unfavorable & Death & 8 & 14.29 \\
& HAMA & 6 & 10.71 \\
& Readmission & 1 & 1.79 \\
\hline
\end{tabular}

antibiotics. The patient had a combination of the ventricular septal defect and rheumatic heart disease. It is shown that the use of penicillin $G$ and amikacin is associated with unfavorable outcome after 4 weeks or less of administration. $(\mathrm{p}=0.007, \alpha=0.05)($ Table 7$)$

Clinical presentation and echocardiographic findings on the time of admission did not correlate with the outcome of children with BCN-IE. (Table 8)

\section{DISCUSSION}

Among 91 patients with infective endocarditis admitted in our institution from 2004 to 2013, 56 (61.54\%) patients had BCN-IE. This finding is quite beyond the usual range of $\mathrm{BCN}-\mathrm{IE}$ seen in various studies, which ranged from $2.5-31 \%$ of all cases. ${ }^{4,5,7}$ However, in reviews of IE in the Philippines, it is within the range of percentage of cases with BCN-IE ranging from $55-72 \%$ with a mean of $60 \% .{ }^{12}$ This may either reflect technical limitations or method in culturing organisms implicated in infective endocarditis, or infection with highly fastidious organisms are more common. ${ }^{1,3,11}$ There is no mention in the literature about specific relations to hospital settings. Nevertheless, a problem in implementing standard procedures or techniques in blood collection (e.g. amount) and culture itself may play a part in decrease yield in blood culture. It was however shown in the study that the problem is not these procedures but rather that the responsible organisms are difficult to culture (fastidious) and thus, appropriate antibiotics against them are best given to the patient. Based on AHA guideline, fastidious organisms that do not grow in routinely used blood culture systems include Bartonella species, Chlamydia species, Coxiella burnetii, Brucella species, Legionella species, Tropheryma whippleii, and non-Candida fungi. Bartonella species, Coxiella burnetii and Brucella species have been the most commonly identified in most series of culture-negative endocarditis caused by fastidious organisms. C. burnetii accounts for $28 \%$ to $37 \%$ of cases, 
Table 7. Comparison of Antibiotic Use, Duration and Outcome of BCN-IE

\begin{tabular}{|c|c|c|c|c|c|}
\hline \multirow{2}{*}{ Antibiotics } & \multicolumn{2}{|c|}{ Favorable } & \multicolumn{2}{|c|}{ Unfavorable } & \multirow{2}{*}{ p-value } \\
\hline & No. of patients $(n=41)$ & Percentage & No. of patients $(n=15)$ & Percentage & \\
\hline \multicolumn{6}{|l|}{ Penicillin G + Amikacin } \\
\hline 4 weeks or less & 5 & 36 & 9 & 64 & \\
\hline 6 weeks & 21 & 91 & 2 & 9 & \\
\hline 8 weeks & 1 & 100 & 0 & 0 & \\
\hline \multicolumn{6}{|c|}{ Penicillin G + Oxacillin + Amikacin } \\
\hline 4 weeks or less & 0 & 0 & 3 & 100 & \\
\hline 6 weeks & 1 & 100 & 0 & 0 & \\
\hline 8 weeks & 0 & 100 & 0 & 100 & \\
\hline \multicolumn{6}{|l|}{ Ceftazidime + Amikacin } \\
\hline 4 weeks or less & 1 & 100 & 0 & 0 & \\
\hline 6 weeks & 2 & 67 & 1 & 33 & \\
\hline 8 weeks & 0 & 100 & 0 & 100 & \\
\hline \multicolumn{6}{|l|}{ Ceftriaxone + Amikacin } \\
\hline 4 weeks or less & 1 & 100 & 0 & 0 & \\
\hline 6 weeks & 1 & 100 & 0 & 0 & \\
\hline 8 weeks & 1 & 100 & 0 & 0 & \\
\hline \multicolumn{6}{|l|}{ Ceftazidime + Oxacillin } \\
\hline 4 weeks or less & 1 & 100 & 0 & 0 & \\
\hline 6 weeks & 0 & 100 & 0 & 100 & \\
\hline 8 weeks & 1 & 100 & 0 & 0 & \\
\hline \multicolumn{6}{|l|}{ Cefepime + Amikacin } \\
\hline 4 weeks or less & 0 & 100 & 0 & 100 & \\
\hline 6 weeks & 2 & 100 & 0 & 0 & \\
\hline 8 weeks & 0 & 100 & 0 & 100 & \\
\hline \multicolumn{6}{|l|}{ Penicillin G + Gentamycin } \\
\hline 4 weeks or less & 1 & 100 & 0 & 0 & \\
\hline 6 weeks & 1 & 100 & 0 & 0 & \\
\hline 8 weeks & 0 & 100 & 0 & 100 & \\
\hline \multicolumn{6}{|l|}{ Vancomycin + Amikacin } \\
\hline 4 weeks or less & 0 & 100 & 0 & 100 & \\
\hline 6 weeks & 1 & 100 & 0 & 0 & \\
\hline 8 weeks & 0 & 100 & 0 & 100 & \\
\hline
\end{tabular}

whereas, Bartonella species, accounts for $12 \%$ to $28 \%$ of cases. ${ }^{9,10}$ T. whipplei causes up to $6 \%$ of cases of culturenegative endocarditis $(9,10,13)$. These organisms cause culture-negative endocarditis due to the requirement for prolonged blood culture incubation for the growth of some strains, while some strains may not grow in blood cultures. Others are usually diagnosed using molecular methods. Antibiotics recommended are the following: ampicillinsulbactam, vancomycin, ciprofloxacin, cefepime, rifampin, ceftriaxone, doxycycline plus gentamicin. Duration of antibiotic use is from 4-6 weeks. ${ }^{3}$ For our patients with BCN-IE, antibiotics used were penicillin $\mathrm{G}$, oxacillin, ceftazidime, ceftriaxone, cefepime and vancomycin plus amikacin/gentamicin. There was a trend to the favorable outcome with those patients given ceftazidime, ceftriaxone, cefepime and vancomycin plus amikacin for 4-6 weeks. Among 38 patients who received penicillin $G$ and amikacin, 22 patients were able to complete 6-8 weeks of treatment and had favorable outcome. Eleven (29\%) had an unfavorable outcome, while 27 (71\%) were discharged well. For BCN-IE, penicillin is not the ideal drug. In this study population however, it was the most frequently used, being the antibiotic of choice against viridans group streptococci, the most common etiologic agents in native valve endocarditis. Use of penicillin and amikacin is significantly associated with unfavorable outcome after 4 weeks or less of administration. This suggests that BCN-IE may be primarily due to fastidious organisms not covered by penicillin and not from a technical error in culturing common organisms.

The most common presenting symptoms are fever followed by dyspnea/orthopnea, easy fatigability and cough. Moreover, edema, pallor and hemiparesis are the most common presenting signs. These findings are consistent with the findings of a similar study conducted by Pena et al and Sabtirul et al. ${ }^{12,14}$ Only a small portion of the patients presented with a new onset heart murmur. This may be in part related to the auscultatory skills of the attending physician. Neurologic and nephrologic sequelae of the disease proved to be common in this population.

More than three fourths of the patients sought consult more than 2 weeks from the onset of their symptoms, as seen also in the study of Werner, et al, in which the mean duration from the first symptom to admission was 23 days. ${ }^{4}$ 
Table 8. Clinical Presentation, Echocardiographic Findings and Outcome of BCN-IE

\begin{tabular}{|c|c|c|c|c|c|c|}
\hline \multirow{2}{*}{\multicolumn{2}{|c|}{ Clinical Presentation }} & \multicolumn{2}{|l|}{ Favorable } & \multicolumn{2}{|c|}{ Unfavorable } & \multirow{4}{*}{$\begin{array}{c}\text { p-value } \\
0.517\end{array}$} \\
\hline & & No. of patients $(n=41)$ & Percentage & No. of patients $(n=15)$ & Percentage & \\
\hline \multirow[t]{2}{*}{ Onset of Symptoms } & $\leq 2$ weeks & 10 & 77 & 3 & 23 & \\
\hline & $>2$ weeks & 31 & 72 & 12 & 28 & \\
\hline \multirow[t]{4}{*}{ Functional Class on Admission } & Class I & 7 & 64 & 4 & 36 & \multirow{4}{*}{0.055} \\
\hline & Class II & 14 & 82 & 3 & 18 & \\
\hline & Class III & 13 & 93 & 1 & 7 & \\
\hline & Class IV & 7 & 50 & 7 & 50 & \\
\hline \multirow[t]{10}{*}{ Predisposing Cardiac Defects } & VSD & 10 & 71 & 4 & 29 & \multirow{10}{*}{0.567} \\
\hline & PDA & 7 & 88 & 1 & 12 & \\
\hline & ASD & 2 & 100 & 0 & 0 & \\
\hline & MR (Congenital) & 0 & 0 & 1 & 100 & \\
\hline & TOF & 4 & 80 & 1 & 20 & \\
\hline & DORV & 1 & 50 & 1 & 50 & \\
\hline & DORV-PVA & 1 & 100 & 0 & 0 & \\
\hline & PVA-VSD & 1 & 100 & 0 & 0 & \\
\hline & RF/RHD & 14 & 74 & 5 & 26 & \\
\hline & None & 1 & 33 & 2 & 67 & \\
\hline \multirow[t]{12}{*}{ Cardiac Structure Involved } & Mitral Valve & 13 & 62 & 8 & 38 & \multirow{12}{*}{0.63} \\
\hline & Tricuspid Valve & 4 & 80 & 1 & 20 & \\
\hline & RVOT & 4 & 100 & 0 & 0 & \\
\hline & Pulmonary Valve (PV) & 3 & 75 & 1 & 25 & \\
\hline & Aortic Valve (native) & 3 & 75 & 1 & 25 & \\
\hline & Pulmonary Artery (PA) & 3 & 100 & 0 & 0 & \\
\hline & Right Atrium & 2 & 100 & 0 & 0 & \\
\hline & Left Atrium & 2 & 100 & 0 & 0 & \\
\hline & Inter-Atrial Septum & 2 & 100 & 0 & 0 & \\
\hline & Interventricular Septum & 2 & 100 & 0 & 0 & \\
\hline & Aortic Valve (Prosthetic) & 0 & 0 & 1 & 100 & \\
\hline & Multiple Locations & 3 & 50 & 3 & 50 & \\
\hline \multirow[t]{3}{*}{ Size of Vegetation } & $<5 \mathrm{~mm}$ & 16 & 76 & 5 & 24 & \multirow{3}{*}{0.929} \\
\hline & $5-10 \mathrm{~mm}$ & 11 & 69 & 5 & 31 & \\
\hline & $>10 \mathrm{~mm}$ & 14 & 74 & 5 & 26 & \\
\hline
\end{tabular}

Half of the patients presented with significant heart failure (NYHA functional class III to IV) and may reflect that most patients will seek to consult only when symptoms are significant. Both the timing of intervention and functional classification upon admission was not significantly correlated with the outcome of patients.

Only a small portion of the patients included in the study had no predisposing heart disease. A third of the patients included in the study had rheumatic fever or rheumatic heart disease. More than half of the patients had congenital heart pathology in which VSD is the most common followed by PDA and TOF. This is in contrast to the study done by Pena et al, in which, rheumatic heart disease was the most common underlying heart disease. ${ }^{12}$ Statistically, the predisposing heart conditions either structural or acquired do not have an influence on the treatment outcome of BCN-IE patients.

The age of patients included varies from 0.04 to 18 years with an average of 10.15 years old. There is a higher incidence of the disease among the early teenage group. This could be attributed to one of the risk factors of IE among teenagers which is dental pathology, although it is beyond the scope of this study. ${ }^{15,16}$ Both male and female are affected and almost equal in incidence which also corresponds to the study of Werner et al. ${ }^{4}$

From the study of Pazin et al., one of the factors that contribute to the negativity of blood culture studies is the recent treatment course of antibiotic. ${ }^{8}$ Only about a fourth of all patients included in the study had previous antibiotic treatment for indications not related to IE. It differs from the study done by Werner et al, in which antibiotic treatment preceded blood culture in $45 \%$ of all episodes of culturenegative endocarditis. ${ }^{4}$

Echocardiography is useful in identifying cardiac lesions that may be potential niduses for endocarditis and provides direct and non-invasive visualization of vegetations especially in $\mathrm{BCN}-\mathrm{IE} .{ }^{17}$ In this study, the size of the vegetations and the presence of valvular dysfunction are not significantly correlated with the outcome of the patient. Mitral valve is the most common location of oscillating mass/vegetation in all BCN-IE patients. This corresponds to the study done by Lamas et al, in which the mitral valve was the most affected valve in $27 \%$ of patients with native valve endocarditis. ${ }^{5}$ This finding coincides with a higher incidence of mitral regurgitation dysfunction among these patients. 


\section{CONCLUSION}

The incidence of $\mathrm{BCN}-\mathrm{IE}$ is high in our institution. The demographic distribution of patients as well as clinical and echocardiographic features are not far different from other related studies and do not have influence on the outcome. Although some BCN-IE may be due to technical inability to culture the common organisms which may explain the favorable outcome in some patients, the trimend to the unfavorable outcome with the use of penicillin suggests the need to target fastidious organisms in $\mathrm{BCN}-\mathrm{IE}$, despite the costs, as recommended in the guidelines. BCN-IE should not be assumed to be due to S. viridans and/or inability of the laboratory to culture this organism. BCN-IE should be assumed to be due to fastidious organisms and be given appropriate antibiotics against these organisms as per the international guidelines.

The data gathered are limited to the objectives of the study and what can be gathered from the chart. Because of this study, BCN-IE patients in UP-PGH are now being given ampicillin-sulbactam (targets fastidious organisms) rather than just penicillin G (targets S. viridans). A followup study on whether there is a decrease in mortality of BCNIE after this intervention is in order. Likewise, mortality and morbidity in all other (culture-positive) IE patients can be the subject of another study.

\section{Recommendation}

Further investigation is warranted to establish the etiologic agents of $\mathrm{BCN}-\mathrm{IE}$, such as serologic tests, polymerase chain reaction (PCR)-based molecular methods, new culture methods and improved media.

\section{Acknowledgement}

The authors would like to thank Roderick Napulan for the statistical support in our study.

\section{Statement of Authorship}

All authors have approved the final version submitted.

\section{Author Disclosure}

All authors declared no conflicts of interest.

\section{Funding Source}

This paper was funded by the corresponding author.

\section{REFERENCES}

1. Allen, H. Driscoll, D. Shaddy, R. Feltes, T. Infective endocarditis and prevention. Moss \& Adams' Heart Disease in Infants, Children, \& Adolescents including the Fetus \& Young Adult, 8th edition. Philadelphia: Lippincott Williams and Wilkins; 2013. pp. 1300-1312.

2. Li JS, Sexton DJ, Mick N, et al. Proposed modifications to the Duke criteria for the diagnosis of infective endocarditis. Clin Infect Dis 2000;30:633-8.

3. Baddour LM, Wilson WR, Bayer AS, et al. Infective Endocarditis Diagnosis, Antimicrobial Therapy, and Management of Complications: A Statement for Healthcare Professionals From the Committee on Rheumatic Fever, Endocarditis, and Kawasaki Disease, Council on Cardiovascular Disease in the Young, and the Councils on Clinical Cardiology, Stroke, and Cardiovascular Surgery and Anesthesia, American Heart Association. Circulation 2005; 111: e394-434.

4. Werner M, Andersson R, Olaison L, et al. A Clinical Study of CultureNegative Endocarditis. Medicine 2003;82: 263-73.

5. Lamas CC, Eykyn SJ. Blood culture negative endocarditis: analysis of 63 cases presenting over 25 years. Heart 2003;89:258-62.

6. Brouqui P, Raoult D. Endocarditis due to rare fastidious bacteria. Clinical Microbiol Rev. 301;14:177-207.

7. Moore J, Millar C. Is the polymerase chain reaction a useful tool or an expensive toy in culture-negative endocarditis? Commun Dis Public Health 2000;3:81-3.

8. Pazin GJ, Saul S. Thompson ME. Blood culture positivity, suppression by outpatient antibiotic therapy in patients with bacterial endocarditis. Arc Internal Med 1982;142:263-8.

9. Houpikian P, Raoult D. Blood Culture-Negative Endocarditis in a Reference Center, Etiologic Diagnosis of 348 Cases. Medicine 2005;84:162-73.

10. Fournier PE, Thuny F, Richet H. Comprehensive Diagnostic Strategy for Blood Culture-Negative Endocarditis: A Prospective Study of 819 New Cases. Clin. Infect. Dis. 2010;51(2):131-40.

11. Oakley CM. The medical treatment of culture-negative infective endocarditis. Eur Heart J. 1995;16 Suppl B:90-3.

12. Pena A.Lim A. Chua J. Guadana Y. Blood culture negative endocarditis: Profile and treatment. Phil J Microbiol Infect Dis 2000;29(2):14-7.

13. Geissdorfer W, Moos V, Moter A, et al. High frequency of Tropheryma whipplei in culture-negative endocarditis. J Clin Microbiol 2012;50:216 -22.

14. Sabtirul ML, Sison MC, Ty ET. Infective Endocarditis in Filipino Pediatric Patients: A Four-Year Review. UP-Philippine General Hospital 2008 (unpublished).

15. Sipes JN. Thompson RL. Hook EW. Prophylaxis of Infective Endocarditis: a re-evaluation. Annu Rev Med 1977;28:371-91.

16. Ito, $\mathrm{H}$. Infective endocarditis and dental procedures: evidence, pathogenesis and prevention. J. Med. Invest. 2006;53:189-98.

17. Rubenson DS, Tucker CR, Stinson EB, et al. The use of echocardiography in diagnosing culture-negative endocarditis. Circulation 1981;64:641-46. 